\title{
ARMSTRONG RELATIONS AND STRONG DEPENDENCIES
}

\author{
VU DUC THI
}

\begin{abstract}
In this paper, the concept of strong scheme is introduced. We prove that the membership problem for strong dependencies is solved by an algorithm in polynomial time.

We give a necessary and sufficient condition for a relation to be Armstrong relation of a given strong scheme.
\end{abstract}

Keyword and Phrases: Relation, relation datamodel, strong scheme, membership problem, SD-relation implication problem, SD-relation equivalence problem, closure, closed set.

\section{RESULTS}

Definition 1. Let $U$ be a nonempty finite set, $R=\left\{h_{1}, \ldots, h_{m}\right\}$ relation over $U$ and $A, U \subseteq U$. We say that $B$ strongly depend on $A$ in $R$ (denote $A \stackrel{s}{\longrightarrow} B$ ) if $\forall h_{i}, h_{j} \in r$ have $h_{i}(a)=h_{j}(a)$ for some $a \in A$ implies $h_{i}(b)=h_{j}(b)$ for all $b \in B$. Let $S_{R}=\{(A, B): A \stackrel{s}{\stackrel{s}{\longrightarrow}} B\}$.

$R_{R}$ is called a full family of strong dependencies of $R$. Where we write $(A, B)$ or $A \rightarrow B$ when $R, s$ are clear from the context.

Definition 2. Let $U=\left\{a_{1}, \ldots, a_{n}\right\}$ be a nonempty finite set attributes. A strong dependency $(S D)$ is a statement of the form $A \rightarrow B$, where $A, B \subseteq U$. The $S D A \rightarrow B$ hold in a relation $R=\left\{h_{1}, \ldots, h_{m}\right\}$ over $U$ if $A \underset{R}{\stackrel{s}{\longrightarrow}} B$. We also say that $r$ satisfies the $S D A \rightarrow B$.

Definition 3. Let $U=\left\{a_{1}, \ldots, a_{n}\right\}$ be a nonempty finite set attributes and $P(U)$ its power set. Let $Y \subseteq P(U) \times P(U)$. We say $Y$ is a s-family if all $A, B, C, D \subseteq U$ and $a \in U$

$S 1:(\{a\},\{a\}) \in Y$

$S 2:(A, B) \in Y,(B, C) \in Y, B$ is no empty set $\Longrightarrow(A, C) \in Y$,

S3 : $(A, B) \in Y, C \subseteq A, D \subseteq B \Longrightarrow(C, D) \in Y$,

$S 4:(A, B) \in Y,(C, D) \in Y \Longrightarrow(A \cup C, B \cap D) \in Y$, 
S5: $(A, B) \in Y,(C, D) \in Y \Longrightarrow(A \cap C, B \cup D) \in Y$.

Clearly, $S_{R}$ is a $s$-family over $U$. It is know [6] that if $Y$ is an arbitrary $s$-family, then there is a relation $R$ over $U$ such that $S_{R}=Y$.

Definition 4. A strong scheme $G$ is a pair $\langle U, S\rangle$, where $U$ is a finite set of attributes, and $S$ a set of $S D s$ over $U$.

Let $S^{+}$be a set of all $S D s$ that can be derived from $S$ by rules Definition 3 .

It can be seen [6] that if $G=\langle U, S\rangle$ is a strong scheme then there is a relation $R$ oves $U$ such that $S_{R}=S^{+}$. Such a relation called Armstrong relation of $G$.

Definition 5. The mapping $F: P(U) \rightarrow P(U)$ is called a strong operation over $U$ if every $a, b \in U$ and $A \in P(U)$ the following properties hold:

(1) $F(0)=U$,

(2) $a \in F(\{a\})$,

(3) $b \in F(\{b\})$ implies $F(\{b\}) \subseteq F(\{a\})$,

(4) $F(A)=\bigcap_{a \in A} F(\{a\})$.

Clearly, if $A \subseteq B$ then $F(B) \subseteq F(A)$, and $F(A) \cup F(B)=F(A \cup B)$. It can be seen that the set $\{F(\{a\}): a \in U\}$ determines the set $\{F(A): A \in P(U)\}$.

Definition 6. Let $R=\left\{h_{i}, \ldots, h_{m}\right\}$ be a relation over $U$ and $A, B \subseteq U$. Then we say $B$ functionally depends on $A$ in $R$ (denote $A \rightarrow B$ ) if

$$
\left.\forall h_{i}, h_{j} \in R\right)(\forall a \in A)\left(h_{i}(a)=h_{j}(a)\right) \Longrightarrow(\forall b \in B)\left(h_{i}(b)=h_{j}(b)\right)
$$

Let $F_{R}=\{(A, B): A, B \subseteq U, A \underset{R}{\stackrel{s}{\longrightarrow}} B\}$

Theorem 1. [11] Let $S$ a s-family over $U$. We define the mapping $F_{s}$ as follows: $F_{s}(A)=\{a \in U:(A,\{a\}) \in S\}$. Then $F_{s}$ is a strong operation over $U$. Conversely, if $F$ is a strong operation over $U$ then there is exactly one s-family over $U$ such that $F_{s}=F$, where $S=\{(A, B): B \subseteq F(A)\}$.

This theorem shows that between $s$-familys and strong operation there exists an one-to-one corresponding.

Lemma 1. Let $G=\langle U, S\rangle$ be a strong sheme, and $A \in P(U)$. We set $A^{+}=\{a \in$ $\left.U: A \rightarrow a \in S^{+}\right\}$. Then $A \rightarrow B \in S^{+}$holds iff $B \subseteq A^{+}$hold.

Proof. If $B \in A^{+}$then by definition of $A^{+}$we have $A \rightarrow\{a\} \forall a \in B$. By S5 $A \rightarrow B$ holds. The converse case is clear. 
Algorithm 1. (Finding $\{a\}^{+}$)

In put: Given a strong scheme $G=\left\langle U, S=\left\{A_{i} \rightarrow B_{i}: i=1, \ldots, m\right\}\right\rangle, a \in U$.

Output: Compute $\{a\}^{+}$.

We compute $\{a\}^{+}$by induction

Step 1: $a \in U$ we set $X^{0}=\{a\}$

Step $i+1$ : If there is an $A_{j} \rightarrow B_{j} \in S$ so that $A_{j} \cap X^{(i)} \neq 0$ and $B \not \subset X^{(i)}$ then $X^{(i+1)}=X^{(i)} \cup\left(\bigcup_{A_{j} \cap X^{(i)} \neq 0 Z} B_{j}\right)$.

In the converse case we set $\{a\}^{+}=X^{1}$. It is easy to see that there is a $t$ such that

$$
\{a\}=\subseteq X^{(1)} \subseteq \cdots \subseteq X^{(t)}=X^{(t+1)}=\cdots \text { and we set }\{a\}^{+}=X^{(t)} .
$$

Proposition 1. For each $a \in U$, Algorithm 1 computes $\{a\}^{+}$.

Proof. We have to prove that an attribute $a^{\prime} \in\{a\}^{+}$iff $a^{\prime} \in X^{(t)}$ holds.

$\Leftarrow$ : we have prove by the induction. It is obvious that $X^{0}=a \in\{a\}^{+}$. We assume that $X^{(1)} \subseteq\{a\}^{+}$, and $a^{\prime} \in X^{(i+1)}-X^{(i)}$. Then there is $A_{j} \rightarrow B_{j} \in S$ so that $\operatorname{acap} X^{(i)} \neq 0, a^{\prime} \in B_{j}-X^{(i)}$. By the inductive hypothesis $\{a\} \rightarrow X^{(i)}$ holds. According to $S 3$ we have $X^{(i)} \rightarrow T=A_{j} \cap X^{(i)}$. hence, $\{a\} \rightarrow T$ holds. By $S 3$ we have $T \rightarrow B_{j}$. By S2 $\{a\} \rightarrow B_{j}$ holds. Consequently, $\{a\} \rightarrow T$ holds, i.e. $a^{\prime} \in\{a\}^{+}$.

$\Rightarrow:$ From rules $S 4$ and $S 5$ we can see that $S D\left\{a_{i 1}, \ldots, a_{i s}\right\} \rightarrow\left\{b_{j 1}, \ldots, b_{j p}\right\}$ is equivalent to the set of $D S s\left\{\left\{a_{i 1}\right\} \rightarrow\left\{b_{j 1}\right\}, \ldots,\left\{a_{i s}\right\} \rightarrow\left\{b_{j p}\right\}\right\}$. Thus, we can assume that the set $S$ only contains $S D s$ form $\{b\} \rightarrow\{c\}$ we call a sequence $S D s$ $\left(f_{1}, \ldots, f_{m}\right)$ is a derivation of a $S D\{a\} \rightarrow B$ iff $f_{m}=\{a\} \rightarrow B$ and for each $i: 1 \leq i \leq m$ one of the following holds:

(1) $f_{i} \in S$ or $f_{i}=\{a\} \rightarrow\{a\}$,

(2) $f_{i}$ is the rusult of applying the $S 2$ to two of $S D s f_{1}, \ldots, f_{i-1}$,

(3) $f_{i}$ is the rusult of applying the $S 3$ to one of $S D s f_{1}, \ldots, f_{i-1}$,

(4) $f_{i}$ is the rusult of applying the $S 4$ or $S 5$ to two of $S D s f_{1}, \ldots, f_{i-1}$,

By induction on the leghth of the (shortest) derivation of $\{a\} \rightarrow B$ we show the converse. Because the set $S$ only contains the $S d s$ form $\{b\} \rightarrow\{c\}$ and by rules $S 4$ we assume (without loss of generality) that in the derivation of a $S D s\{a\} \rightarrow B f_{1}=\{a\} \rightarrow\left\{a_{j}\right\}$, where $a_{j} \in U$ and each $f_{i}$ has the form $\{a\} \rightarrow C$ or $f_{i} \in S$. We assume that $a^{\prime} \in\{a\}^{+}$and $a^{\prime} \in B$. If the sequence $\left\{f_{1}, \ldots, f_{m}\right\}$ of $\{a\} \rightarrow B$ has $m=1$ then it is obvios that $B \subseteq X^{(t)}$. Now we consider the devation $\left\{f_{1}, \ldots, f_{i+1}\right\}$ of $\{a\} \rightarrow B$. If $f_{i+1}=\{a\} \rightarrow B=\left\{a^{\prime}\right\}$ then by the construction of $X$ we obtain $a^{\prime} \in X^{(t)}$. It is easy to see that if $f_{i+1}$ is the result of applying $S 2$ 
to $f_{p}$ and $f_{p}(1 \leq p \leq i, 1 \leq q \leq i)$ or is result of applying $S 3$ to $f_{s}(1 \leq s \leq i)$ then by the induction hypothesis and the construction of $X^{(t)} B \subseteq X^{(t)}$ holds. If $f_{i+1}$ is the result of applying $S 5(S 4)$ two $S D s f_{p}=\{a\} \rightarrow C$ and $f_{q}=\{a\} \rightarrow D$ $(p, q \leq i)$ then by the induction hypothesis there are $X^{(l)}$ and $X^{(h)}$ such that $C \subseteq X^{(l)}, D \subseteq X^{(h)}$. We set $s=\max (l, h)$, then $B=C \cup D \subseteq X^{(s)}$ and $B=C \cap D \subseteq X^{(l)}$. Thus, $B \subseteq X^{(t)}$, i.e. $a^{\prime} \in X^{(t)}$. The proposition is proved.

It can be seen that Algorithm 1 is polynomial time in the $|U|,|G|$, and $A^{+}=$ $\cap\{a\}^{+}$. Thus, the following proposition is clear. $a \in A$

Proposition 2. (The membership problem)

Let $G=\langle U, S\rangle$ be a strong scheme over $U$, and $A \rightarrow B$ is a strong dependency, then there is a pollynomial time algrithm deciding whether $A \rightarrow B \in S^{+}$.

Definition 7. Let $R=\left\{h_{1}, \ldots, h_{m}\right\}$ be a relation over $U$. $R_{E}$ is the equality set of $R$, i.e. $E_{i j}=\left\{a \in U: h_{i}(a)=h_{j}(a)\right\}$ and $E_{R}=\left\{E_{i j}: 1 \leq \subset \leq j \leq m\right\}$. We set $E(a)=\bigcap_{a \in E_{i j}} E_{i j}$ if there is a such $E_{i j}$ in the converse case set $E(a)=U$, where $a \in U$.

Denote $E_{R}^{*}=\{E(a): a \in U\} . E_{R}^{*}$ is called the attribute-equality set of $R$.

Clearly, we can compute $E_{R}^{*}$ in polynmial time in the size of $R$.

Theorem 2. Let $G=\langle U, S\rangle$ be a strong scheme, $R=\left\{h_{1}, \ldots, h_{m}\right\}$ a relation over $U$ and $E_{R}^{*}$ in the attribute equality set of $R$.

Then necessary and sufficient condition for $R$ to be Armtrong relation of strong sheme $G$ is for each $a \in U:\{a\}^{+}=E(a)$, where $E(a) \in E_{R}^{*}$.

Proof. We set $A_{R}^{+}=\{a \in U: A \stackrel{s}{\stackrel{R}{\longrightarrow}}\{a\}\}$.

Now we show that $\{a\}_{R}^{+}=E(a)$ for each $a \in U$. By definition of strong dependency we know that for any $a \in U:\{a\} \underset{R}{\stackrel{s}{\longrightarrow}} B$ iff $\{a\} \underset{R}{\stackrel{f}{\longrightarrow}} B$.

Denote $H=\left\{E_{i j}: a \in E_{i j}\right\}$ It can be seen that if $H=\emptyset$ then $\{a\} \underset{R}{\stackrel{s}{\longrightarrow}} U$.

We assume that $H \neq \emptyset$. It is easy to see that if $H=E_{R}$ holds, then by the definition of $E(a)$ and definiton of strong dependency $\{a\} \underset{R}{\stackrel{s}{\longrightarrow}} E(a)$ holds.

If $H \subseteq E_{R}$ holds then for $E_{i j} \notin H$ we obatain $h_{i}(a) \neq h_{j}(a)$. Consequently, $\{a\} \underset{R}{\stackrel{s}{\longrightarrow}} E(a)$.

It can seen that $\forall E: E \supset E(a)$ we obtain $\{a\} \underset{R}{\stackrel{s}{\longrightarrow}} E(a)$.

According to the definition of $\{a\}_{R}^{+}$we have $\{a\}_{R}^{+}=E(a)$. 
Clearly, by Theorem 1 we can see that $S_{R}=S^{+}$iff for each $a \in U:\{a\}^{+}=$ $\{a\}_{R}^{+}$holds. Thus, if $S_{R}=S^{+}$holds then $\{a\}^{+}=E(a)$ for all $a \in U$.

Conversely, according to Theorem 1 and base on $\{a\}_{R}^{+}=E(a)$ for all $a \in U$ we obain $S_{R}=S^{+}$. The theorem is proved.

\section{REFERENCES}

1. Arstrong W. W. Dependency Structutes of Database Relationships. Information Processing 74, Holland publ. Co., (1974) 580-583.

2. Beeri C., Dowd M, Fagin R., Staman R., On the Structure of Armstrong relations for Functional Dependencies. J. ACM, 31 (1) (1984) 30-46.

3. Beeri C., Bernstein P.A., Compuiational problems related to the design of normal form relational schemas. ACM Trans. on Database syst., 4 (1) (1979) 30-59.

4. Burosch G., Demetrovics J., Katona G.O.H., The poset of closures as a model of changing databases, Order 4 (1987) 127-142.

5. Czedli $\mathrm{G}$. On the dependencies in the relational model of data. J. EIK, 17 (2-3) (1981) 103-112.

6. Demetrovics J., Relacios adatmodell logikai es strukturalis vizsgalata. MTA-SZTAKI Tanulmanyok, Budapest, 114 (1980) 1-97.

7. Demetrovics J., Gyepesi G., On the functional dependency and generalizations of it. Acta Cybernetica Hungary, Vol. 3 (1983) 295-305.

8. Demetrovics J., Thi. V.D., Relations and minimal keys. Acta Cybernetica Hungary VIII/3 (1988) 279-285.

10. Thi V.D., Minimal keys and Antikeys. Acta Cybernetica Hungary, Tom. 7, Fasc. 4 (1986) 361-371.

11. Thi. V.D. Strong dependencien and s-semilattices. Acta Cybernatica, VIII (2) (1987) 195202.

12. Thi V. V., Logical dependencies and irredundant relations. Computers and Artificial Intelligence, 7 (1988) 165-184.

13. Gottlob G., Libkin L., Investigations on Armtrong ralations, dependency inference, and excluded functional dependencies. Acta Cybernetica Hungary, Tom. 9 Fasc. 4 (1990) 385-402.

14. Mannila H., Raiha K. J., Algorithms for inferring functional dependencies. Proc. of the thirteenth international conferebce on very large data bases, Brighton, September, 1987.

15. Lucchesi C.L., Osborn S.L., Candidate keys for relations. J. Comput. Syst. Scien., 17 (2) (1978) 270-279.

16. Demetrovics J., Thi V.D., On the algorithms for generating Armstrong relations and for inferring functional depencies in the relation datamodel. Computer and Mathematics with Applications, Gred Britain, 26 (4) (1993) 43-55.

17. Demetrovich J., Thi V. D., Some relults about normal form for functional dependency in the relational. Discrete Applied Mathematics, 69 (1966) 61-74.

Institute of Information Technology

National Centre for Sciences and Technology of Vietnam.

Received: April 90, 1996 